\title{
miR-29a-3p directly targets Smad nuclear interacting protein 1 and inhibits the migration and proliferation of cervical cancer HeLa cells
}

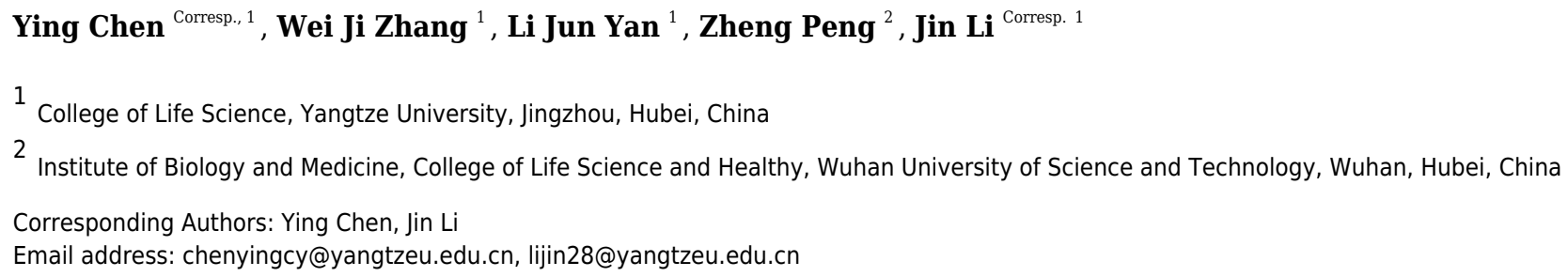

Smad nuclear interacting protein 1 (SNIP1) is a nuclear protein and involved in essential biological processes. MicroRNAs are effective regulators of tumorigenesis and cancer progression via targeting multiple genes. In present study, we aimed to investigate the function of SNIP1 and identify novel miRNA-SNIP1 axis in the development of cervical cancer. The results showed for the first time that silencing of the SNIP1 gene inhibited the migration and proliferation in HeLa cells significantly. Bioinformatics analysis and dual luciferase reporter assay demonstrated that miR-29a-3p could target 3'UTR of SNIP1 directly. The mRNA and protein expression levels of SNIP1 were negative regulated by miR-29a-3p according to the RT-qPCR and Western blot analysis, respectively. Furthermore, functional studies showed that over-expression of miR-29a-3p restrained HeLa cells migration and proliferation, and the mRNA expression of SNIP1 downstream genes (HSP27, c-Myc, and cyclin D1) were down-regulated by miR-29a-3p. Together, we concluded that miR-29a-3p suppressed the migration and proliferation in HeLa cells by directly targeting SNIP1. The newly identified miR-29a-3p/SNIP1 axis could provide new insight into the development of cervical cancer. 
1 miR-29a-3p directly targets Smad nuclear interacting

2 protein 1 and inhibits the migration and proliferation

3 in cervical cancer HeLa cells

4

5 Ying Chen ${ }^{1}$, Weiji Zhang ${ }^{1}$, Lijun Yan ${ }^{1}$, Peng Zheng ${ }^{2}$, Jin Li ${ }^{1}$

6

$7 \quad{ }^{1}$ College of Life Science, Yangtze University, Jingzhou, Hubei, China

$8 \quad{ }^{2}$ College of life and healthy, Wuhan University of Science and Technology, Wuhan, Hubei,

9 China

10

11 Corresponding Author:

12 Ying Chen ${ }^{1}, \mathrm{Jin}_{\mathrm{Li}^{1}}$

13 No. 266 Jingmi Road, Jingzhou, Hubei, 434025, People's Republic of China.

14 Email address: chenyingcy@yangtzeu.edu.cn, lijin28@yangtzeu.edu.cn 


\section{Abstract}

27 Smad nuclear interacting protein 1 (SNIP1) is a nuclear protein and involved in essential

28 biological processes. MicroRNAs are effective regulators of tumorigenesis and cancer

29 progression via targeting multiple genes. In present study, we aimed to investigate the function

30 of SNIP1 and identify novel miRNA-SNIP1 axis in the development of cervical cancer. The

31 results showed for the first time that silencing of the SNIP1 gene inhibited the migration and

32 proliferation in HeLa cells significantly. Bioinformatics analysis and dual luciferase reporter

33 assay demonstrated that miR-29a-3p could target 3'UTR of SNIP1 directly. The mRNA and

34 protein expression levels of SNIP1 were negative regulated by miR-29a-3p according to the RT-

35 qPCR and Western blot analysis, respectively. Furthermore, functional studies showed that over-

36 expression of miR-29a-3p restrained HeLa cells migration and proliferation, and the mRNA

37 expression of SNIP1 downstream genes (HSP27, c-Myc, and cyclin D1) were down-regulated by

38 miR-29a-3p. Together, we concluded that miR-29a-3p suppressed the migration and proliferation

39 in HeLa cells by directly targeting SNIP1. The newly identified miR-29a-3p/SNIP1 axis could

40 provide new insight into the development of cervical cancer.

\section{Introduction}

42 Cervical cancer is the fourth most prevalent malignancy and remains the major cause of cancer 43 death among females worldwide (Bray et al., 2018).Despite there are considerable 44 improvements of treatment strategies in the therapy of cervical cancer, the overall 5-year 45 survival rates of patients remain poor for metastasis (Forouzanfar et al., 2011; Tewari et al., 46 2014; $L i, W u$, \& Cheng, 2016). Therefore, understanding the pathogenesis and progression of 47 cervical cancer is quite necessary and may facilitate identification of effective therapeutic 48 targets for cervical cancer treatment. 
49

50

51

52

53

54

55

56

57

58

Smad nuclear interacting protein 1 (SNIP1), as an evolutionarily conserved nuclear protein, is involved in essential biological processes such as cell proliferation (Roche et al,. 2004; Fujii et al., 2006), small RNA biogenesis (Yu et al., 2008), DNA damage response (Chen et al., 2018), and several signaling pathways (Kim et al., 2000; Kim et al., 2001). In patients with non-small cell lung cancer or tongue squamous cell carcinoma, SNIP1 might be a reliable prognostic indicator (Liang et al., 2011; Jeon et al., 2013). Recently, SNIP1 was considered to be targeted by microRNA-335 and involved in osteosarcoma proliferation and metastasis (Xie et al., 2019). However, the exact role of SNIP1 in the development of cervical cancer remains obscure. MicroRNAs (miRNAs) are a group of non-coding RNAs with 19-25 nucleotides and have closely relationships with the occurrence and progression of human cancers (Romano et al., 2017). MicroRNAs regulate gene expression mainly through binding to the 3 '-untranslated region (UTR) of target mRNAs (Bartel, 2004). As oncogene or tumor suppressor in different cancers, miRNAs play essential roles in basic biological processes such as cell proliferation, apoptosis, differentiation, migration and invasion (Bartel, 2004; Pardini et al., 2018).

Accumulating studies have shown that miR-29a was abnormally expressed in various cancers, including cervical cancer (Pei, Lei, \& Liu, 2016; Yang et al., 2017; Gong et al., 2019). However, the detailed role and underlying mechanism of miR-29a in cervical cancer is still largely unclear.

In this research, we investigated the biological role of SNIP1 in the progression of cervical cancer. Furthermore, we predicted and demonstrated that miR-29a-3p inhibited the transcription and protein expression of SNIP1 by targeting 3'UTR directly, and suppressed the proliferation and migration of cervical cancer cells.

\section{Materials \& Methods}




\section{Cell culture}

73 The human cervical cancer HeLa cells were purchased from American Type Culture Collection

74 (Manassas, VA), and maintained in Dulbecco's modified Eagle's medium (Hyclone, USA)

75 containing 10\% fetal bovine serum (Gibco, USA) under a humidified environment with $5 \% \mathrm{CO}_{2}$ 76 at $37^{\circ} \mathrm{C}$.

\section{Transfection}

78 Three small interfering RNAs (siRNAs) targeting SNIP1 (siSNIP1-330, siSNIP1-871, siSNIP1-

79 1059) were purchased from GenePharma (Suzhou, China). The miR-29a-3p mimics and negative 80 control (NC) were manufactured by RiboBio (Guangzhou, China). All transfections were 81 performed using siRNA-Mate reagent (GenePharma, China) in accordance with the instruction 82 manual. The cells were collected after $48 \mathrm{~h}$ of transfection for further experiments.

\section{Quantitative RT-PCR (RT-qPCR)}

84 Total RNA was harvested from HeLa cells by EZNA Total RNA Kit (Omega BioTek, USA).

85 The first-strand cDNA was generated using HiScript II Q RT SuperMix (Vazyme, China). RT-

86 qPCR was performed to quantify relative RNA levels using ChamQ Universal SYBR qPCR

87 Master Mix (Vazyme, China) on a CFX96 Touch (Bio-rad, USA). The $2^{-\Delta \Delta C t}$ method was used to 88 measure the relative expression level, and GAPDH served as the internal reference. The primers 89 used for RT-qPCR were presented in Table1.

\section{Western blot analysis}

91 The cultured cells were lysed with RIPA buffer (Beyotime, China). The protein concentration

92 was quantified by Enhanced BCA Assay kit (Beyotime, China). Total protein samples were

93 separated with 10\% SDS-PAGE gel and transferred onto PVDF membrane (Millipore, USA).

94 After blocked with 5\% skim milk, the membrane was probed with primary antibodies against 
95 SNIP1 (1:1000, Proteintech, USA) and GAPDH (1:20000, Proteintech, USA) at $4{ }^{\circ} \mathrm{C}$ overnight

96 and followed by incubation with secondary antibodies (1:4000, Beyotime, China). Blots were

97 visualized by BeyoECL Plus Kit (Beyotime, China) and scanned with a ChemiDoc XRS imaging

98 system (Bio-Rad, USA).

\section{Dual luciferase reporter assay}

100 To determine the binding affinity between SNIP1 and miR-29a-3p, the recombinant psiCHECK-

1012 vectors (Promega, USA) with the wild type (WT) or mutant of SNIP1 gene 3'-UTR were

102 constructed. Then, the recombinant vectors (WT or mutant) and miR-29a-3p mimics (or NC)

103 were co-transfected in HeLa cells. Dual-Luciferase Reporter Assay system (Promega, USA) was

104 used to calculate the luciferase activity after transfection according to the manual instruction.

105 Scratch assay

106 Transfected HeLa cells were cultured in 6-well plates until the confluence reached 100\%. Then,

107 a sterile pipet tip was used to scrape on the bottom of culture plates. Cell migration was observed

108 at $12 \mathrm{~h}$ under an inverted microscope (Olympus, Japan) and images were captured for each

109 sample. The scratch area was measured and analyzed by ImageJ software (NIH, MD, USA).

110 Cell Counting Kit-8 assay

111 Hela cells (3000 cells/well) were seeded into 96-well plates after transfection, and cultured for

11224 h, 48 h, 72 h and 96 h. Fresh medium with $10 \%$ CCK-8 (Genview Scientific, AUS) was

113 mixed carefully, and the absorbance values of $450 \mathrm{~nm}$ wavelength were detected at least three

114 times by a spectrophotometric plate reader (Hitachi, Japan).

115 Transwell migration assay

116 Transfected cells $\left(5 \times 10^{4}\right)$ were placed into the top chamber of the transwell inserts (Corning,

117 USA) and maintained with serum-free medium. Then the lower chamber was filled with 
118 complete medium. And the migrated cells were fixed and stained 24 hours later. Finally, the cells

119 stained in more than three visual fields were randomly selected and photographed with an

120 inverted microscope (Olympus, Japan) and counted.

121 Statistical analysis

122 Statistical analysis was carried out using the GraphPad Prism 8 (GraphPad Software, SanDiego, 123 USA) program. All results were presented as mean \pm standard error (SD) of three independent 124 experiments. Comparison among multiple groups was assessed by Student's $t$ test. A statistically 125 significant difference was defined as $P<0.05$.

126

127

\section{Results}

\section{Knockdown of SNIP1 reduced migration and proliferation in cervical cancer cells}

To address the biological role of SNIP1 in the progression of cervical cancer, three small interference RNAs (siRNAs) targeting SNIP1 were synthesized and transfected into HeLa cells. Among them, siSNIP1-330 showed the best silencing effect (Fig. 1A and B), which was subsequently chosen for further analysis. After transfected with siSNIP1-330, the wound area ratio increased (Fig. 1C) and the number of migrated cells decreased (Fig. 1D), which demonstrated the migration of HeLa cells was suppressed. Furthermore, the cell proliferation rate declined after transfection for $48 \mathrm{~h}$ (Fig. 1E). In addition, the mRNA expression levels of several migration-related genes (MMP9, VIM, MAPK1, N-cadherin and E-cadherin) and proliferationrelated genes (CDK2) in HeLa cells (Wang \& Chen, 2019) can also be downregulated or upregulated (Fig. 1F). Hence, SNIP1 knockdown could reduce migration and proliferation in cervical cancer HeLa cells.

\section{SNIP1 was directly targeted by miR-29a-3p}


140 To further confirm the exact miRNA that can directly target SNIP1, three different

141 bioinformation tools starBase, TargetScan and miRanda, were performed to calculate the

142 possibility scores. Intersection of these three sets showed that there were 12 candidate miRNAs

143 which target SNIP1(Fig. 2A), and miR-29a-3p was chosen for the highest score (Table 2).

144 Moreover, the expression relationship between miR-29a-3p and SNIP1 is negative correlation

$145(P<0.05)$ in cervical squamous cell carcinoma and endocervical adenocarcinoma (CESC)

146 samples from starBase (Fig. 2B, Table 2). Compared with the control group, the luciferase

147 activity was inhibited significantly in HeLa cells when co-transfected with SNIP1 wild type 3'-

148 UTR vector and miR-29a-3p mimics (Figs. 2C and D). Furthermore, both mRNA and protein

149 expression levels of SNIP1 in HeLa cells were declined after transfection with miR-29a-3p

150 mimics (Figs. 2E and F).Taken together, these results demonstrated that miR-29a-3p targeted

151 SNIP1 via directly binding its 3' UTR region and negatively regulated SNIP1 expression in

152 cervical cancer.

153 MiR-29a-3p inhibited migration and proliferation in cervical cancer cells

154 To evaluate the regulatory roles of miR-29a-3p in HeLa cells, the scratch assay and transwell

155 assay for migration were performed. As results shown in Figs. 3A and C, transfection with miR-

156 29a-3p mimics restrained migration in HeLa cells. Moreover, miR-29a-3p mimics also

157 significantly decreased the relative cell viability in HeLa cells (Fig. 3B). Additionally, miR-29a-

$1583 p$ also regulated the mRNA expression levels of genes associated with migration or

159 proliferation in HeLa cells (Fig. 3D). Therefore, these data indicated that miR-29a-3p inhibited

160 migration and proliferation in cervical cancer cells.

161 MiR-29a-3p regulated the mRNA expression of SNIP1 downstream genes 
162 To investigate whether miR-29a-3p would have effects on the downstream of SNIP1, the mRNA

163 levels of these downstream genes (HSP27, c-Myc and Cyclin D1) (Fujii et al., 2006; Bracken et

164 al., 2008; Zhu et al., 2010) were detected by RT-qPCR after transfection with miR-29a-3p

165 mimics or siSNIP1-330. The data suggested that miR-29a-3p and knockdown of SNIP1 both

166 reduced the expression of those genes significantly (Fig. 4A).

\section{Discussion}

168 SNIP1 is a transcription regulator contains a nuclear localization sequence, and plays a key role 169 in tumor development and progression (Kim et al., 2000; Kim et al., 2001; Fujii et al., 2006;

170 Bracken et al., 2008). Over-expression of SNIP1 promoted cell invasion and migration in 171 osteosarcoma cells (Xie et al., 2019). And the knockdown of SNIP1 restrained the anchorage172 independent growth of lung cancer cells (Jeon et al., 2013). However, the function of SNIP1 in 173 cervical cancer development is poorly understood. In our analysis, the migration and 174 proliferation of cervical cancer cells was significantly suppressed after siRNA-mediated 175 silencing of SNIP1 (Figs. 1C, D and E). These results suggested that SNIP1 was involved in the 176 development of HeLa cells as an oncogene.

177 As a member of the miR-29 family, miR-29a is considered to play a crucial role in the 178 regulation of multiple cancers (Wang et al., 2018). It was up-regulated and promoted epithelial179 mesenchymal transition, migration and invasion in breast cancer cells (Wu et al., 2019). In 180 contrast, other studies have shown that miR-29a was down-regulated and inhibited the 181 progression of cancers such as colon cancer (Shi et al., 2019), non-small cell lung cancer (Hu et 182 al., 2016) and adenocarcinoma (Zhang et al., 2018). Previous studies have shown that miR-29a 183 was low expression in cervical cancer, as a tumor suppressor, miR-29a can target multiple genes, 184 such as CDC42, HSP47, SIRT1 and DNMT1 (Park et al., 2009; Yamamoto et al., 2013; Gong et 
185 al., 2019; Nan et al., 2019). Nonetheless, the detailed biological function of miR-29a in cervical 186 cancer has not yet been completely revealed. In this research, we showed for the first time that 187 SNIP1 was targeted by miR-29a-3p in cervical HeLa cells. The results indicated that miR-29a-3p 188 could down-regulate SNIP1 expression levels (Figs. 2E and F), and the direct binding site of 189 SNIP1 mRNA 3'UTR was confirmed by dual luciferase reporter assay (Fig. 2D). Furthermore, 190 our data also supported previous reports (Yamamoto et al., 2013), indicating that miR-29a-3p can 191 suppress the migration and proliferation of HeLa cells (Figs. 3A, B and C). Sharing the same 192 seed region in miR-29 family (miR-29a/b/c), we inferred that SNIP1 may also be targeted by 193 other miR-29s.

194 It has been reported that SNIP1 could improve the transcriptional activity of c-Myc (Fujii et 195 al., 2006), and regulate the stability of Cyclin D1 mRNA (Bracken et al., 2008). Otherwise, 196 SNIP1 could down-regulate the transcription of HSP27 (Zhu et al., 2010). These downstream 197 genes (c-Myc, Cyclin D1 and HSP27) regulated by SNIP1 are considered to be closely 198 associated with cell proliferation and migration (Evan et al., 1994; Zhu et al., 2010; Li et al., 2012; Pestell, 2013). In this research, miR-29a-3p decreased the mRNA expression levels of $c$ 200 Myc, Cyclin D1 and HSP27 in HeLa cells as an upstream regulator (Fig. 4A). Therefore, miR$20129 a-3 p$ may mediate the regulation of cell proliferation and migration in cervical cancer cells via 202 downstream genes of SNIP1 (Fig. 4B). Further study is required to illustrate the underlying 203 mechanism of miR-29a-3p/SNIP1 pathway in cervical cancer oncogenesis.

204 In addition, this research has some defects in the following aspects. Although it was confirmed 205 that miR-29a-3p can target SNIP1 to inhibit the migration and proliferation of HeLa cells, 206 whether over-expression of SNIP1 could supplement the inhibitory effect of miR-29a-3p should 207 be further observed. It was more convinced to detect the protein levels related to migration, 
208 proliferation as well as downstream of SNIP1, while the mRNA levels were reduced markedly 209 by miR-29a-3p or SNIP1 siRNA in HeLa cells. In this study, we have used only one cell line to 210 verify the function of miR-29a-3p/SNIP1, more cell lines and more in vitro and in vivo 211 experiments are needed to be carried out. Finally, miR-590-3p is worth exploring as miR-29a-3p 212 for the Pearson correlation analysis suggested that miR-590-3p was negatively correlated with 213 SNIP1 in CESC tissues $(r=-0.149, P=0.00883)$ (Table 2$)$.

\section{Conclusions}

215 In conclusion, miR-29a-3p suppressed the migration and proliferation of cervical cancer cells by 216 directly targeting SNIP1, and could also down-regulate the mRNA expression of SNIP1 217 downstream genes such as c-Myc, Cyclin D1 and HSP27. The newly identified miR-29a$2183 \mathrm{p} / \mathrm{SNIP} 1$ axis may provide new insights into the understanding of the progression of cervical 219 cancer, and represent an effective treatment target for cervical cancer.

\section{References}

221 Bartel D. P. 2004. MicroRNAs: genomics, biogenesis, mechanism, and function. Cell 116(2): $222 \quad 281-297$ https://doi.org/10.1016/s0092-8674(04)00045-5

223 Bracken, C. P., Wall, S. J., Barré, B., Panov, K. I., Ajuh, P. M., \& Perkins, N. D. 2008.

224 Regulation of cyclin D1 RNA stability by SNIP1. Cancer research 68(18): 7621-7628

225 https://doi.org/10.1158/0008-5472.CAN-08-1217

226 Bray, F., Ferlay, J., Soerjomataram, I., Siegel, R. L., Torre, L. A., \& Jemal, A. 2018. Global 227 cancer statistics 2018: GLOBOCAN estimates of incidence and mortality worldwide for 36 228 cancers in 185 countries. CA: a cancer journal for clinicians 68(6): 394-424

229 https://doi.org/10.3322/caac.21492 
230 Chen, L. L., Lin, H. P., Zhou, W. J., He, C. X., Zhang, Z. Y., Cheng, Z. L., Song, J. B., Liu, 231 P., Chen, X. Y., Xia, Y. K., Chen, X. F., Sun, R. Q., Zhang, J. Y., Sun, Y. P., Song, L., Liu, 232 B. J., Du, R. K., Ding, C., Lan, F., Huang, S. L., Zhou, F., Liu, S., Xiong, Y., Ye, D., \& 233 Guan, K. L. 2018. SNIP1 Recruits TET2 to Regulate c-MYC Target Genes and Cellular DNA 234 Damage Response. Cell reports 25(6): 1485-1500 e4.

235 https://doi.org/10.1016/j.celrep.2018.10.028

236 Evan, G., Harrington, E., Fanidi, A., Land, H., Amati, B., \& Bennett, M. 1994. Integrated 237 control of cell proliferation and cell death by the c-myc oncogene. Philosophical transactions of 238 the Royal Society of London. Series B, Biological sciences 345(1313): 269-275

239 https://doi.org/10.1098/rstb.1994.0105

240 Forouzanfar, M. H., Foreman, K. J., Delossantos, A. M., Lozano, R., Lopez, A. D., Murray, 241 C. J., \& Naghavi, M. 2011. Breast and cervical cancer in 187 countries between 1980 and 2010: 242 a systematic analysis. Lancet (London, England) 378(9801): 1461-1484

243 https://doi.org/10.1016/S0140-6736(11)61351-2

244 Fujii, M., Lyakh, L. A., Bracken, C. P., Fukuoka, J., Hayakawa, M., Tsukiyama, T., Soll, S. 245 J., Harris, M., Rocha, S., Roche, K. C., Tominaga, S. I., Jen, J., Perkins, N. D., Lechleider, 246 R. J., \& Roberts, A. B. 2006. SNIP1 is a candidate modifier of the transcriptional activity of c247 Myc on E box-dependent target genes. Molecular cell 24(5): 771-783

248 https://doi.org/10.1016/j.molcel.2006.11.006

249 Gong, Y., Wan, J. H., Zou, W., Lian, G. Y., Qin, J. L., \& Wang, Q. M. 2019. MiR-29a

250 inhibits invasion and metastasis of cervical cancer via modulating methylation of tumor

251 suppressor SOCS1. Future oncology (London, England) 15(15): 1729-1744

252 https://doi.org/10.2217/fon-2018-0497 
253 Hu, Z., Cui, Y., Zhou, Y., Zhou, K., Qiao, X., Li, C., \& Wang, S. 2016. MicroRNA-29a plays 254 a suppressive role in non-small cell lung cancer cells via targeting LASP1. OncoTargets and 255 therapy 9: 6999-7009. https://doi.org/10.2147/OTT.S116509

256 Jeon, H. S., Choi, Y. Y., Fukuoka, J., Fujii, M., Lyakh, L. A., Song, S. H., Travis, W. D., 257 Park, J. Y., \& Jen, J. 2013. High expression of SNIP1 correlates with poor prognosis in non258 small cell lung cancer and SNIP1 interferes with the recruitment of HDAC1 to RB in vitro. Lung 259 cancer (Amsterdam, Netherlands) 82(1): 24-30 https://doi.org/10.1016/j.lungcan.2013.07.015

260 Kim, R. H., Wang, D., Tsang, M., Martin, J., Huff, C., de Caestecker, M. P., Parks, W. T., 261 Meng, X., Lechleider, R. J., Wang, T., \& Roberts, A. B. 2000. A novel smad nuclear 262 interacting protein, SNIP1, suppresses p300-dependent TGF-beta signal transduction. Genes \& 263 development 14(13): 1605-1616

264 Kim, R. H., Flanders, K. C., Birkey Reffey, S., Anderson, L. A., Duckett, C. S., Perkins, N. 265 D., \& Roberts, A. B. 2001. SNIP1 inhibits NF-kappa B signaling by competing for its binding to 266 the $\mathrm{C} / \mathrm{H} 1$ domain of CBP/p300 transcriptional co-activators. The Journal of biological chemistry 267 276(49): 46297-46304 https://doi.org/10.1074/jbc.M103819200

268 Li, H., Wu, X., \& Cheng, X. 2016. Advances in diagnosis and treatment of metastatic cervical 269 cancer. Journal of gynecologic oncology 27(4): e43. https://doi.org/10.3802/jgo.2016.27.e43 270 Li, Q., An, J., Liu, X., Zhang, M., Ling, Y., Wang, C., Zhao, J., \& Yu, L. 2012. SNIP1: a 271 new activator of HSE signaling pathway. Molecular and cellular biochemistry 362(1-2): 1-6 272 https://doi.org/10.1007/s11010-011-1120-y

273 Liang, X., Zheng, M., Jiang, J., Zhu, G., Yang, J., \& Tang, Y. 2011. Hypoxia-inducible 274 factor-1 alpha, in association with TWIST2 and SNIP1, is a critical prognostic factor in patients 
275 with tongue squamous cell carcinoma. Oral oncology 47(2): 92-97

276 https://doi.org/10.1016/j.oraloncology.2010.11.014

277 Nan, P., Niu, Y., Wang, X., \& Li, Q. 2019. MiR-29a function as tumor suppressor in cervical 278 cancer by targeting SIRT1 and predict patient prognosis. OncoTargets and therapy 12: 69172796925 https://doi.org/10.2147/OTT.S218043

280 Pardini, B., De Maria, D., Francavilla, A., Di Gaetano, C., Ronco, G., \& Naccarati, A. 2018. 281 MicroRNAs as markers of progression in cervical cancer: a systematic review. BMC cancer 282 18(1): 696 https://doi.org/10.1186/s12885-018-4590-4

283 Park, S. Y., Lee, J. H., Ha, M., Nam, J. W., \& Kim, V. N. 2009. miR-29 miRNAs activate p53 284 by targeting p85 alpha and CDC42. Nature structural \& molecular biology 16(1): 23-29 285 https://doi.org/10.1038/nsmb.1533

286 Pei, Y. F., Lei, Y., \& Liu, X. Q. 2016. MiR-29a promotes cell proliferation and EMT in breast 287 cancer by targeting ten eleven translocation 1. Biochimica et biophysica acta 1862(11): 21772882185 https://doi.org/10.1016/j.bbadis.2016.08.014

289 Pestell R. G. 2013. New roles of cyclin D1. The American journal of pathology 183(1): 3-9 290 https://doi.org/10.1016/j.ajpath.2013.03.001

291 Roche, K. C., Wiechens, N., Owen-Hughes, T., \& Perkins, N. D. 2004. The FHA domain 292 protein SNIP1 is a regulator of the cell cycle and cyclin D1 expression. Oncogene 23(50): 81852938195 https://doi.org/10.1038/sj.onc.1208025

294 Romano, G., Veneziano, D., Acunzo, M., \& Croce, C. M. 2017. Small non-coding RNA and 295 cancer. Carcinogenesis 38(5): 485-491 https://doi.org/10.1093/carcin/bgx026

296 Shi, X., Valizadeh, A., Mir, S. M., Asemi, Z., Karimian, A., Majidina, M., Safa, A., \& Yosefi, 297 B. 2020. miRNA-29a reverses P-glycoprotein-mediated drug resistance and inhibits proliferation 
298 via up-regulation of PTEN in colon cancer cells. European journal of pharmacology 880:

299 173138. https://doi.org/10.1016/j.ejphar.2020.173138

300 Tewari, K. S., Sill, M. W., Long, H. J., 3rd, Penson, R. T., Huang, H., Ramondetta, L. M.,

301 Landrum, L. M., Oaknin, A., Reid, T. J., Leitao, M. M., Michael, H. E., \& Monk, B. J.

302 2014. Improved survival with bevacizumab in advanced cervical cancer. The New England

303 journal of medicine 370(8): 734-743 https://doi.org/10.1056/NEJMoa1309748

304 Wang, J. Y., \& Chen, L. J. 2019. The role of miRNAs in the invasion and metastasis of cervical 305 cancer. Bioscience reports 39(3): BSR20181377. https://doi.org/10.1042/BSR20181377

306 Wang, J. Y., Zhang, Q., Wang, D. D., Yan, W., Sha, H. H., Zhao, J. H., Yang, S. J., Zhang,

307 H. D., Hou, J. C., Xu, H. Z., He, Y. J., Hu, J. H., Zhong, S. L., \& Tang, J. H. 2018. MiR-29a:

308 a potential therapeutic target and promising biomarker in tumors. Bioscience reports $\mathbf{3 8 ( 1 )}$

309 BSR20171265. https://doi.org/10.1042/BSR20171265

310 Wu, Y., Shi, W., Tang, T., Wang, Y., Yin, X., Chen, Y., Zhang, Y., Xing, Y., Shen, Y., Xia,

311 T., Guo, C., Pan, Y., \& Jin, L. 2019. miR-29a contributes to breast cancer cells epithelial-

312 mesenchymal transition, migration, and invasion via down-regulating histone H4K20

313 trimethylation through directly targeting SUV420H2. Cell death \& disease 10(3): 176.

314 https://doi.org/10.1038/s41419-019-1437-0

315 Xie, Y., Deng, H., Wei, R., Sun, W., Qi, Y., Yao, S., Cai, L., Wang, Y., \& Deng, Z. 2019.

316 Overexpression of miR-335 inhibits the migration and invasion of osteosarcoma by targeting

317 SNIP1. International journal of biological macromolecules 133: 137-147

318 https://doi.org/10.1016/j.ijbiomac.2019.04.016

319 Yang, Y. L., Wang, F. S., Li, S. C., Tiao, M. M., \& Huang, Y. H. 2017. MicroRNA-29a

320 Alleviates Bile Duct Ligation Exacerbation of Hepatic Fibrosis in Mice through Epigenetic 
321 Control of Methyltransferases. International journal of molecular sciences 18(1): 192

322 https://doi.org/10.3390/ijms18010192

323 Yamamoto, N., Kinoshita, T., Nohata, N., Yoshino, H., Itesako, T., Fujimura, L.,

324 Mitsuhashi, A., Usui, H., Enokida, H., Nakagawa, M., Shozu, M., \& Seki, N. 2013. Tumor-

325 suppressive microRNA-29a inhibits cancer cell migration and invasion via targeting HSP47 in

326 cervical squamous cell carcinoma. International journal of oncology 43(6): 1855-1863

327 https://doi.org/10.3892/ijo.2013.2145

328 Yu, B., Bi, L., Zheng, B., Ji, L., Chevalier, D., Agarwal, M., Ramachandran, V., Li, W.,

329 Lagrange, T., Walker, J. C., \& Chen, X. 2008. The FHA domain proteins DAWDLE in

330 Arabidopsis and SNIP1 in humans act in small RNA biogenesis. Proceedings of the National

331 Academy of Sciences of the United States of America 105(29): 10073-10078

332 https://doi.org/10.1073/pnas.0804218105

333 Zhang, Y., He, S., Mei, R., Kang, Y., Duan, J., Wei, R., Xiang, C., Wu, Y., Lu, X., Cai, Z., 334 \& Xiong, L. 2018. miR-29a suppresses IL 13 induced cell invasion by inhibiting YY1 in the 335 AKT pathway in lung adenocarcinoma A549 cells. Oncology reports 39(6): 2613-2623.

336 https://doi.org/10.3892/or.2018.6352

337 Zhu, Z., Xu, X., Yu, Y., Graham, M., Prince, M. E., Carey, T. E., \& Sun, D. 2010. Silencing

338 heat shock protein 27 decreases metastatic behavior of human head and neck squamous cell

339 cancer cells in vitro. Molecular pharmaceutics 7(4): 1283-1290

340 https://doi.org/10.1021/mp100073s 
Table $\mathbf{1}$ (on next page)

Primers for quantitative RT-PCR 
Table 1 Primers for quantitative RT-PCR

\begin{tabular}{ccc} 
Gene & Forward 5'-3' & Reverse 5'-3' \\
\hline SNIP1 & GCTTTGTGGACCAGGTGTTT & TGTACAGTCACGGGCTTGAG \\
cyclin D1 & TTTGTTGTGTGTGCAGGGAG & TTTCTTCTTGACTGGCACGC \\
CDK2 & TGAAGATGGACGGAGCTTGT & ACTGGAGGAGAGGGTGAGAT \\
MMP9 & GCGTCTTCCCCTTCACTTTC & ATAGGGTACATGAGCGCCTC \\
MAPK1 & GAACTTCTGCAACCCCACTG & CAGCCGCAGTTATAAGCAGG \\
$N$-cadherin & GACAATGCCCCTCAAGTGTT & CCATTAAGCCGAGTGATGGT \\
E- cadherin & CGGACGATGATGTGAACACC & TTGCTGTTGTGCTTAACCCC \\
HSP27 & AGTGGTCGCAGTGGTTAGG & TCCTTGGTCTTGACCGTCAG \\
c-Myc & AACACACAACGTCTTGGAGC & GCACAAGAGTTCCGTAGCTG \\
GIM & AGCTAACCAACGACAAAGCC & TTGCGTTCAAGGTCAAGACG \\
\hline & CGACCACTTTGTCAAGCTCA & AGGGGTCTACATGGCAACTG \\
\hline
\end{tabular}




\section{Table 2 (on next page)}

Potential miRNAs binding SNIP1 and co-expression analysis for the miRNA-SNIP1 in CESC

Note: CESC, cervical squamous cell carcinoma and endocervical adenocarcinoma. 1, analysis from TargetScan7.2 (http://www.targetscan.org/vert_72/). 2, analysis from starBase v3.0 pancancer analysis(http://starbase.sysu.edu.cn/panMirCoExp.php). 


\begin{tabular}{|c|c|c|c|c|c|}
\hline \multirow[b]{3}{*}{ miRNA } & \multicolumn{4}{|c|}{ Table 2 Potential miRNAs binding SNIP1 and } & \multirow[b]{3}{*}{$p$-value ${ }^{2}$} \\
\hline & \multicolumn{2}{|l|}{ Position } & \multicolumn{2}{|l|}{ context ++ score } & \\
\hline & in the $\mathrm{UTR}^{1}$ & seed match ${ }^{1}$ & percentile $^{1}$ & $r^{2}$ & \\
\hline hsa-miR-29a-3p & 293-299 & 7 mer-m8 & 96 & -0.165 & $3.91 \mathrm{E}-03$ \\
\hline hsa-miR-542-3p & $836-842$ & 7 mer-m8 & 94 & -0.057 & $3.19 \mathrm{E}-01$ \\
\hline hsa-miR-384 & $314-321$ & 8 mer & 91 & 0.000 & $1.00 \mathrm{E}+00$ \\
\hline hsa-miR-520d-5p & $361-367$ & 7 mer-m8 & 90 & 0.023 & $6.91 \mathrm{E}-01$ \\
\hline hsa-miR-371a-5p & $504-510$ & 7 mer-m8 & 89 & -0.034 & $5.59 \mathrm{E}-01$ \\
\hline hsa-miR-520a-3p & $535-541$ & 7 mer-1A & 88 & 0.098 & $8.66 \mathrm{E}-02$ \\
\hline hsa-miR-520b & $535-541$ & $7 \mathrm{mer}-1 \mathrm{~A}$ & 87 & 0.012 & $8.29 \mathrm{E}-01$ \\
\hline hsa-miR-520c-3p & $535-541$ & $7 \mathrm{mer}-1 \mathrm{~A}$ & 87 & 0.017 & 7.64E-01 \\
\hline hsa-miR-362-3p & $1058-1064$ & 7 mer- $1 \mathrm{~A}$ & 66 & -0.034 & $5.59 \mathrm{E}-01$ \\
\hline hsa-miR-590-3p & $1191-1197$ & 7 mer-m8 & 59 & -0.149 & $8.83 \mathrm{E}-03$ \\
\hline hsa-miR-107 & $2140-2146$ & $7 \mathrm{mer}-1 \mathrm{~A}$ & 46 & 0.013 & $8.15 \mathrm{E}-01$ \\
\hline hsa-miR-217 & $2143-2149$ & 7 mer-1A & 46 & -0.083 & $1.50 \mathrm{E}-01$ \\
\hline
\end{tabular}

2 Note:

3 CESC, cervical squamous cell carcinoma and endocervical adenocarcinoma.

4 1, analysis from TargetScan7.2 (http://www.targetscan.org/vert_72/).

5 2, analysis from starBase v3.0 pan-cancer analysis

6 (http://starbase.sysu.edu.cn/panMirCoExp.php). 


\section{Figure 1}

Knockdown of SNIP1 inhibited HeLa cells migration and proliferation

(A-B) RT-qPCR and Western blot analysis of SNIP1 expression in different siRNAs transfected HeLa cells. (C-D) Scratch assay and transwell analysis were performed to determine the migration of HeLa cells transfected with siSNIP1-330 or siNC, respectively. (E) Cell viability was measured using the CCK-8 assay after knockdown SNIP1 in HeLa cells at 24 h, 48 h, and $72 \mathrm{~h}$. (F) RT-qPCR analysis of migration-related genes and proliferation-related genes in HeLa cells infected with siSNIP1-330 or siNC, respectively. GAPDH was used as internal control. Each experiment were repeated three times, $* P<0.05$, ${ }^{*} P<0.01$. 
A

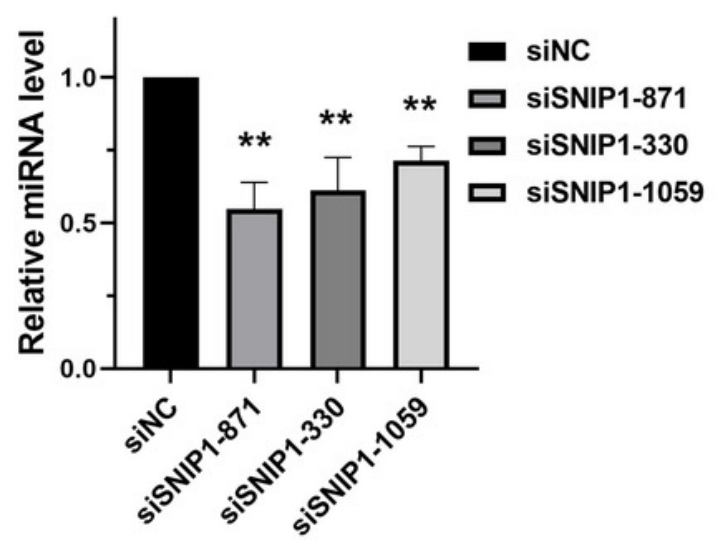

C
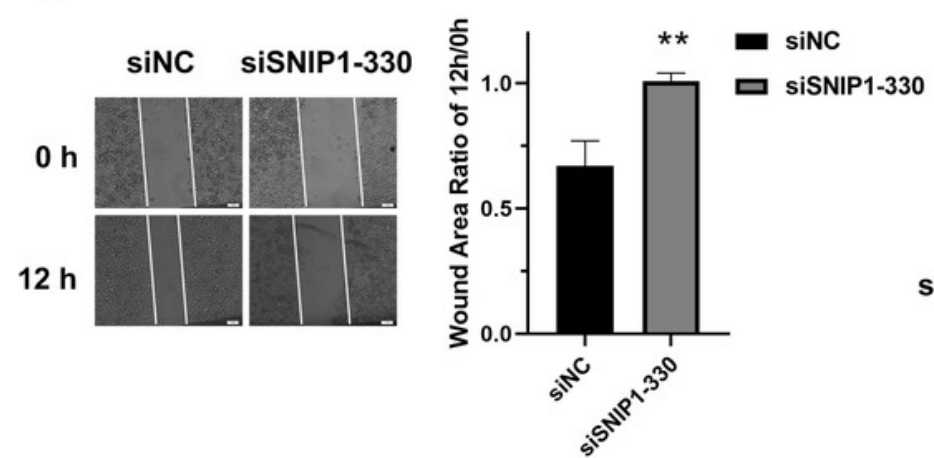

E

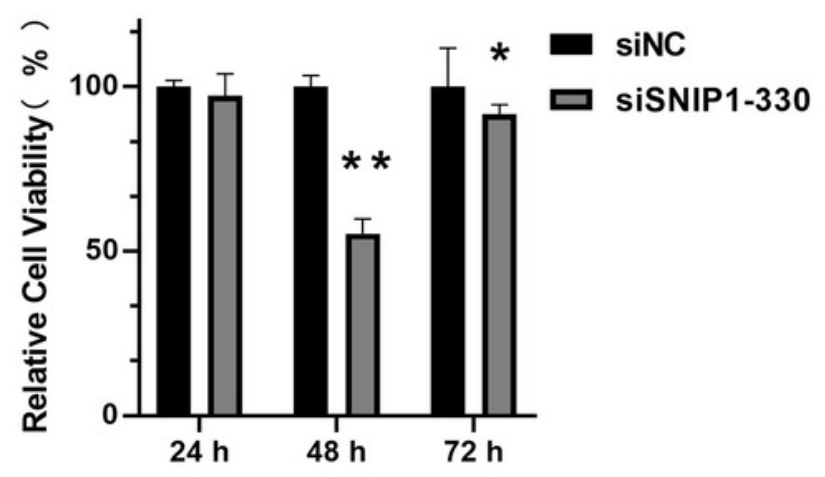

B

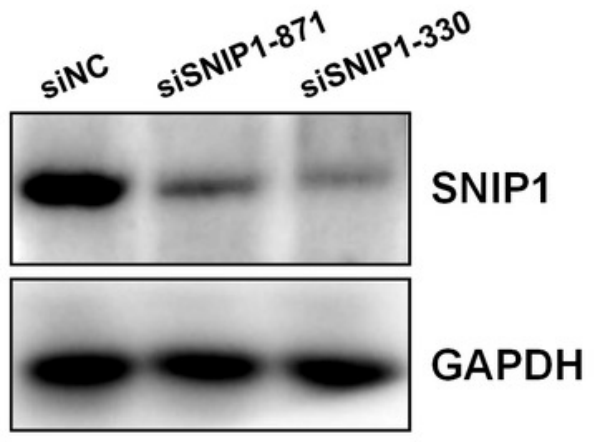

D
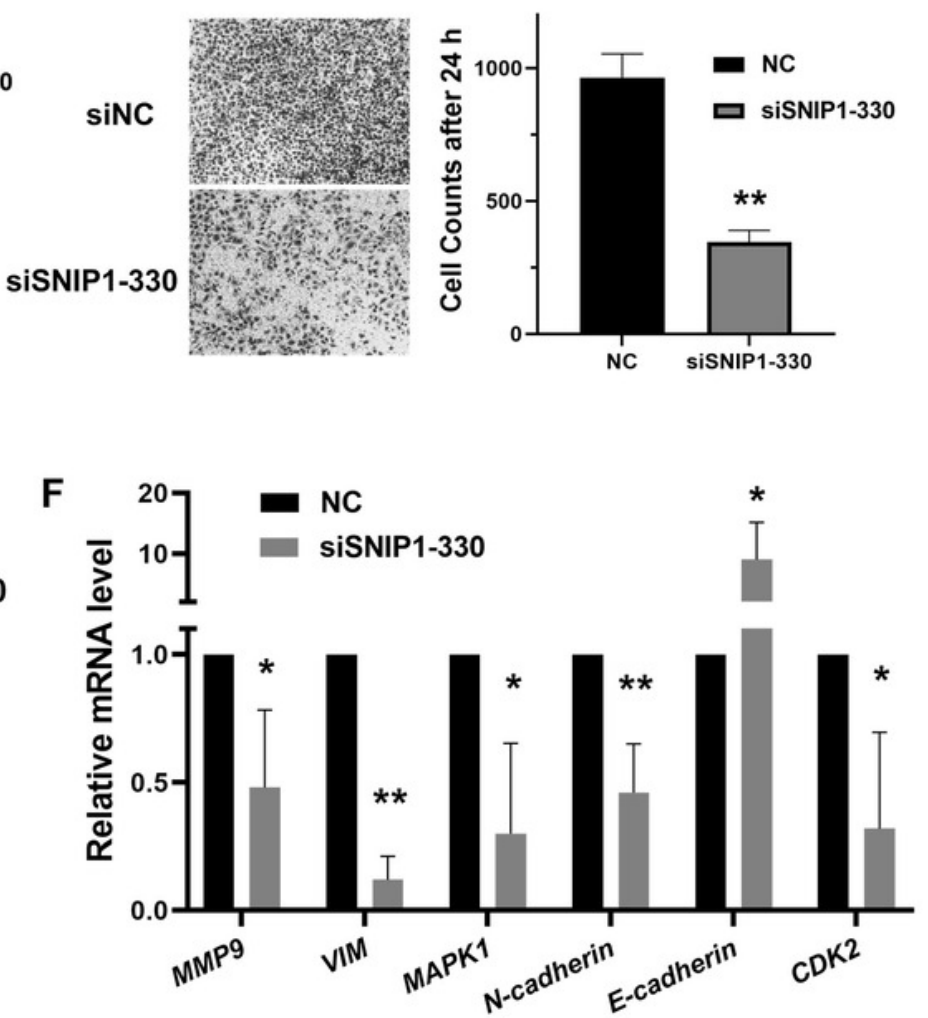


\section{Figure 2}

miR-29a-3p directly targets SNIP1 in HeLa cells

(A) Potential miRNAs binding SNIP1 predicted in starBase, TargetScan and miRanda. (B) Correlation between miR-29a-3p with SNIP1 in CESC samples on the basis of starBase v3.0 pan-cancer analysis. (C) Graphical presentation of putative binding sites for miR-29a-3p in the wild type and mutant type 3'UTR of SNIP1. (D) Dual-luciferase reporter assay was performed following transfection with miR-29a-3p mimics or NC mimics using psi-check2 luciferase vector including the SNIP1 WT 3'UTR or the mutant 3'UTR. (E) After transfection of miR-29a-3p mimics or NC mimics in HeLa cells, SNIP1 mRNA expression were detected by RTqPCR. (F) SNIP1 protein expression was measured by Western blotting after transfection. GAPDH served as the internal control. $* P<0.05$. 
A

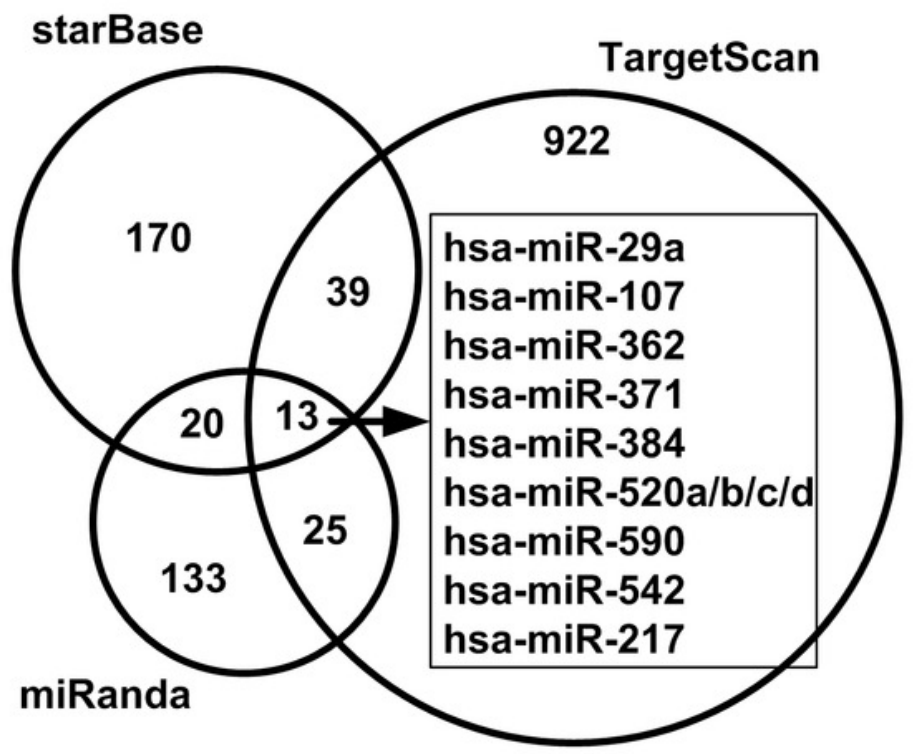

C

5' ...UguUgugguUUUAguUggugCUU... Position 293-299

| | | | || | | |

3' AUUGGCUAAAGUCUACCACGAU hsa-miR-29a-3p

$3^{\prime}$

AUUGGCUAAAGUCUACCACGAU SNIP1 3' UTR Mut
B

hsa-miR-29a-3p vs. SNIP1, 306 samples (CESC) Data Source: starBase v3.0 project

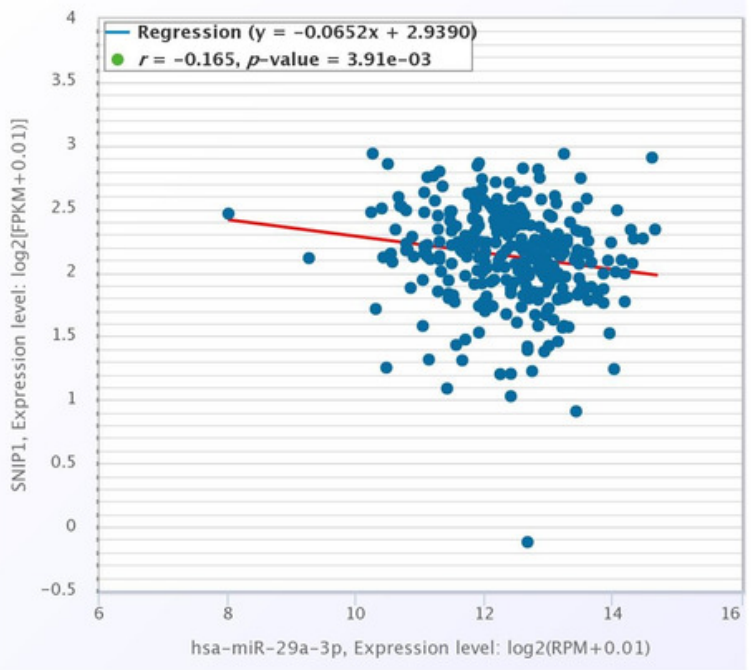

D

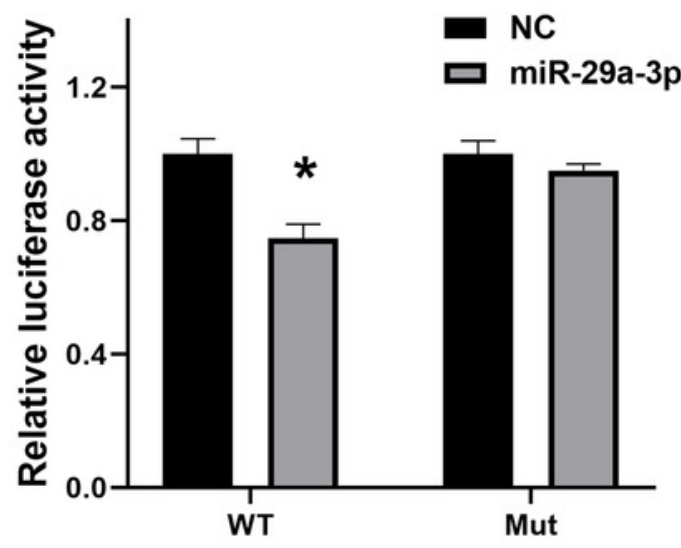

$\mathbf{F}$

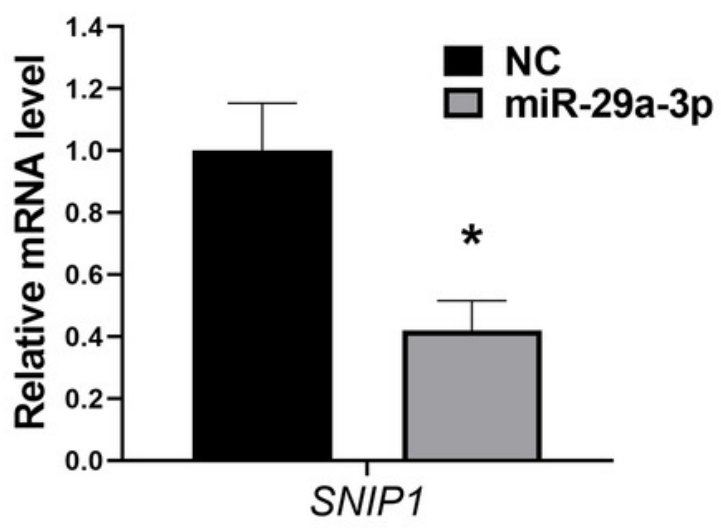

NC miR-29a-3p

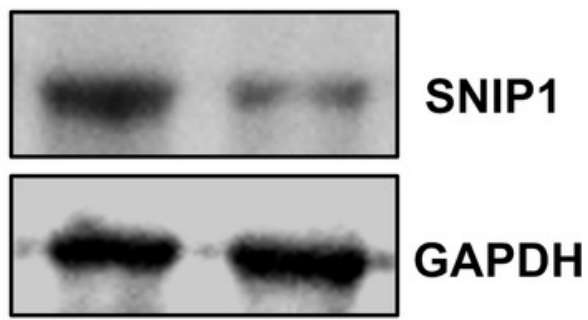




\section{Figure 3}

MiR-29a-3p inhibited HeLa cells migration and proliferation.

(A) Cell scratch assay to evaluate HeLa cells migration after transfection of miR-29a-3p mimics or NC, respectively. The ratio of area $12 \mathrm{~h} / 0 \mathrm{~h}$ was measured by Image J. (B) Cell viability was measured after transfection of miR-29a-3p mimics or NC. (C) The effects of miR-29a-3p on migration were determined by transwell assay in HeLa cells. (D) RT-qPCR analysis of migration-related genes and proliferation-related genes in HeLa cells transfected with miR-29a-3p mimics or NC. $* P<0.05, * * P<0.01$.
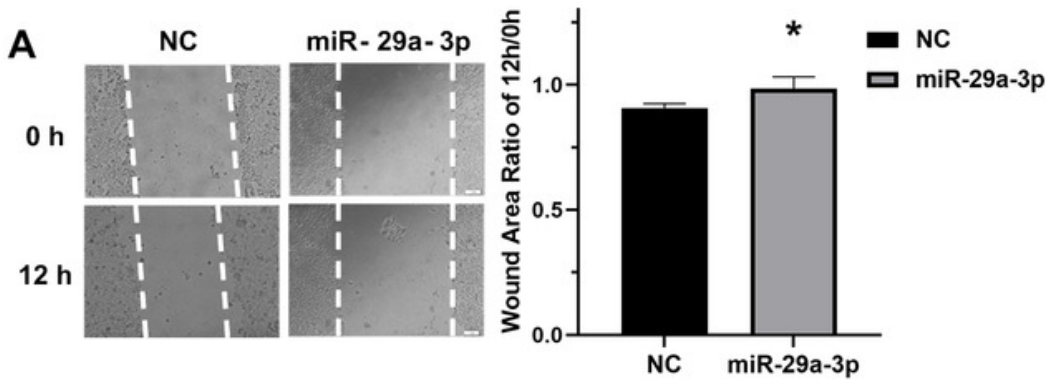

C

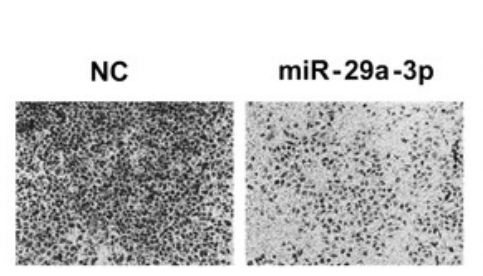

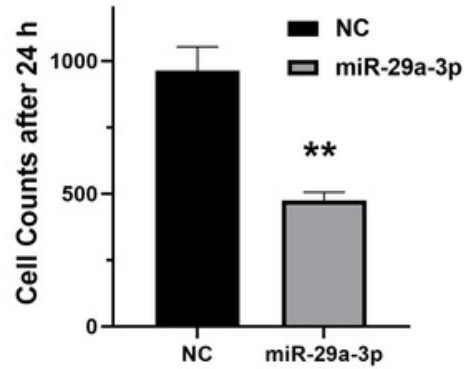

B
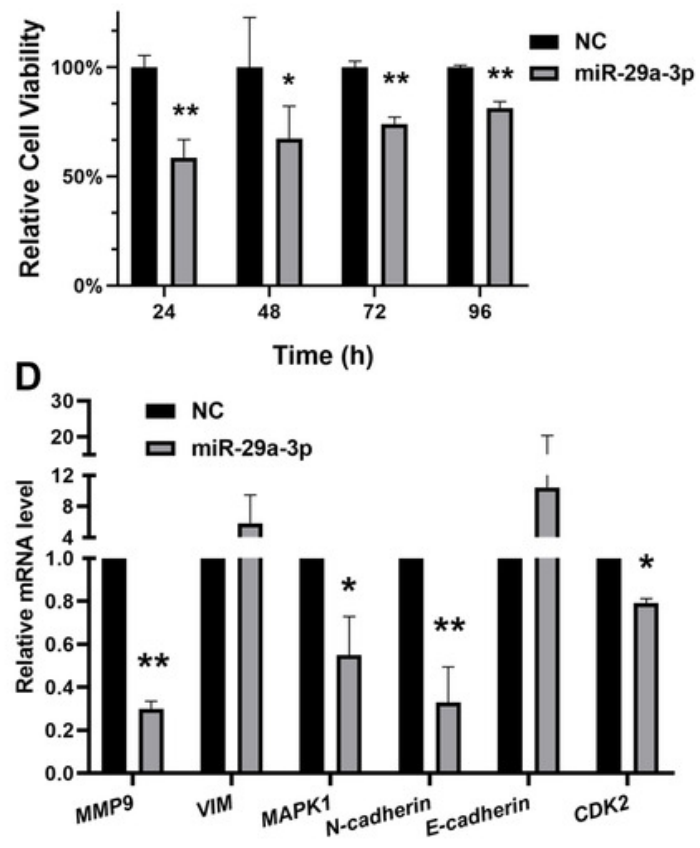
Figure 4

MiR-29a-3p regulated the downstream genes of SNIP1.

(A) The relative mRNA level of downstream genes of SNIP1 (HSP27, c-Myc and cyclin D1) in HeLa cells transfected with miR-29a-3p mimics, siSNIP1-330 or NC were determined by RTqPCR. (B) Schematic diagram of miR-29a-3p and SNIP1 regulate migration and proliferation in HeLa cells. $* P<0.05, * * P<0.01$.

A

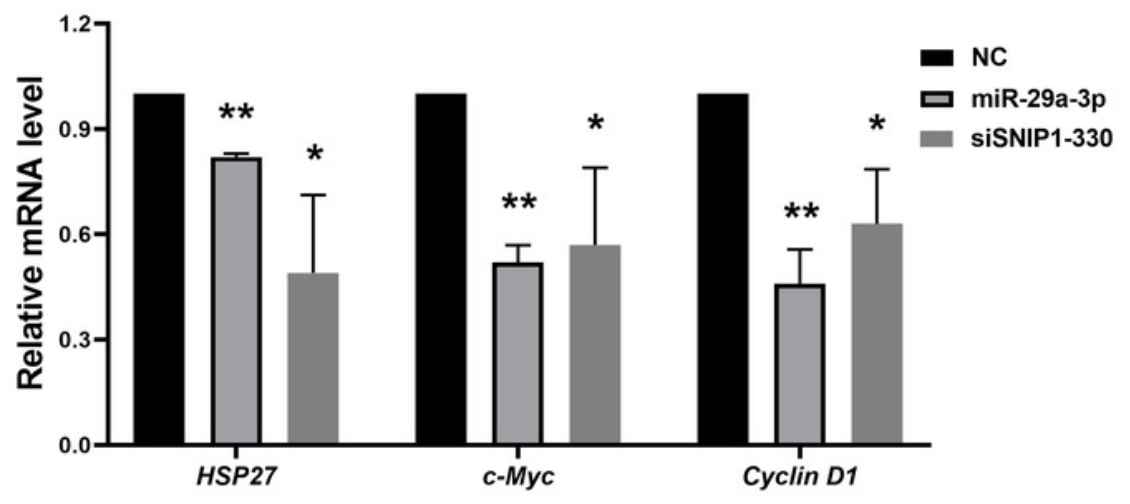

B

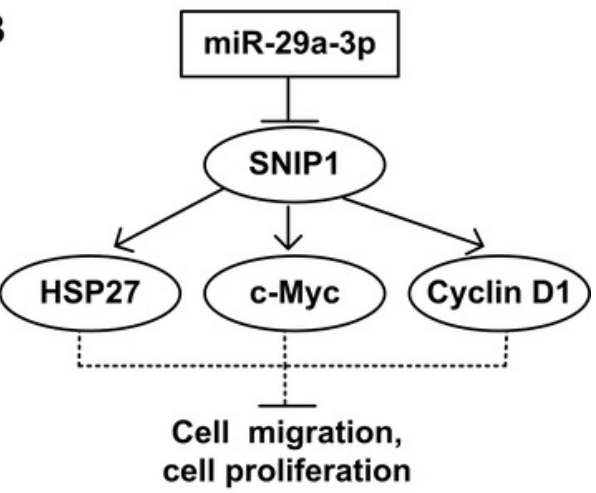

